\title{
Broad HIV neutralization by a vaccine-induced cow antibody
}

\author{
R Stanfield ${ }^{1}$ \\ ${ }^{1}$ The Scripps Research Institute La Jolla, CA \\ robyn@scripps.edu
}

Potent broadly neutralizing antibodies to HIV have been very challenging to elicit by vaccination in wild-type animals. Using $\mathrm{x}$-ray crystallography, cryo-electron microscopy and site-directed mutagenesis, we have analyzed the mode of binding of a potent bnAb (NC-Cow1) elicited in cows by immunization with the HIV gp140 Envelope trimer BG505 SOSIP.664. The exceptionally long (60 residues) third complementarity determining region of the NC-Cow1 heavy chain forms a knob-shaped mini-domain on an extended stalk that navigates through the dense glycan shield on the HIV envelope trimer to target a small footprint at the gp120 CD4 receptor binding site with no contact of the other CDRs to the rest of the Env trimer. These findings illustrate how an unusual vaccine-induced cow bnAb to HIV Env can neutralize with high potency and breadth.

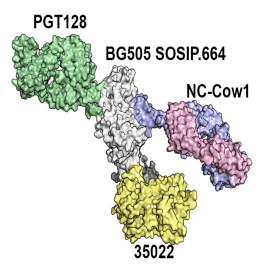

Figure 1

Acta Cryst. (2020). A76, a113 